PROCEEDINGS OF THE

AMERICAN MATHEMATICAL SOCIETY

Volume 125, Number 10, October 1997, Pages 3069-3073

S 0002-9939(97)04175-0

\title{
HYPERSPACES AND CONES
}

\author{
SERGIO MACÍAS \\ (Communicated by James West)
}

\begin{abstract}
We characterize locally connected continua $X$ for which its hyperspace of subcontinua, $\mathcal{C}(X)$, has finite dimension and is homeomorphic to the cone of a continuum $Z$.
\end{abstract}

\section{INTRODUCTION}

During the history of hyperspaces several people have studied those continua $X$ for which its hyperspace of subcontinua $\mathcal{C}(X)$ is homeomorphic to its cone $\mathcal{K}(X)$; see for example [D-R], [I], [N1], [N2, chapter VIII], [R1], and [R2]. In his book [N2], Nadler also considers the case when the hyperspace $\mathcal{C}(X)$ of a continuum $X$ is homeomorphic to the cone $\mathcal{K}(Z)$ of a continuum $Z$. Nadler ([N2, p. 333]) says that he can prove that "if $X$ is a locally connected continuum for which its hyperspace $\mathcal{C}(X)$ has finite dimension and is homeomorphic to the cone of a continuum $Z$, then $X$ and $Z$ must be arcs or circles". However, we prove the following result:

Theorem 3. If $X$ is a simple $n$-od, then $\mathcal{C}(X)$ is homeomorphic to the cone $\mathcal{K}(Z)$ of a continuum $Z$.

We also prove the following theorem:

Theorem 4. Let $X$ be a locally connected continuum whose hyperspace of subcontinua, $\mathcal{C}(X)$, has finite dimension. If $\mathcal{C}(X)$ is homeomorphic to the cone, $\mathcal{K}(Z)$, over a continuum $Z$, then $X$ is an arc, a simple closed curve, or a simple $n$-od.

Definitions. If $Z$ is a topological space and $A \subset Z$, then the closure of $A$ in $Z$ is denoted by $\mathrm{Cl}_{Z}(A)$, or by $\mathrm{Cl}(A)$ if there is no confusion, its interior by $\operatorname{Int}_{Z}(A)$, and its boundary by $\partial_{Z}(A)$. If $(Y, d)$ is a metric space, then given $A \subset Y$ and $\varepsilon>0$, the open ball around $A$ of radius $\varepsilon$ is denoted by $\mathcal{V}_{\varepsilon}(A)$; we will write $\mathcal{V}_{\varepsilon}(y)$ for $\mathcal{V}_{\varepsilon}(\{y\})$. If $A$ is a subset of $Y$, then $\operatorname{diam}(A)=\sup \left\{d\left(a_{1}, a_{2}\right) \mid a_{1}, a_{2} \in A\right\}$. The set of positive integers is denoted by $\mathbb{N}$.

A continuum is a nonempty, compact, connected, metric space. A subcontinuum of a continuum $X$ is a continuum contained in $X$. By a graph we mean a continuum which can be written as the union of finitely many arcs, any two of which are either disjoint or intersect only in one or both of their end-points. By a segment of a graph $X$ we shall always mean one of those arcs or a simple closed curve. The end-points of the segments of $X$ are called vertices of $X$. Given a point $x \in X$ and

Received by the editors November 19, 1995.

1991 Mathematics Subject Classification. Primary 54B20.

Key words and phrases. Hyperspace, continuum, cone.

(C)1997 American Mathematical Society 


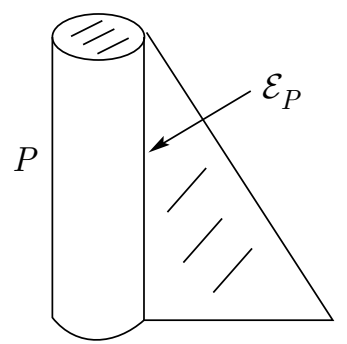

Figure 1

a natural number $n$, the order of $X$ at $x$, denoted $\operatorname{ord}_{x} X$, is $n$ provided that for every $\varepsilon>0$, there exists an open set $U$ of $X$ containing $x$ with $\operatorname{diam}(U)<\varepsilon$ such that $\partial_{X}(U)$ consists of exactly $n$ points ([N3, Chapter 9]). For each point $v \in X$, we have either $\operatorname{ord}_{v} X=1$ if $v$ is an end-point of $X$, or $\operatorname{ord}_{v} X \geq 2$ otherwise. If $\operatorname{ord}_{v} X \geq 3$, then $v$ is called a ramification point of $X$. By a simple $n$-od $(n \geq 3)$ we mean a graph $X$ with only one ramification point and exactly $n$ segments without circles. A simple 3-od will be called a simple triod.

Given a continuum $X$, the hyperspace of nonempty subcontinua of $X$ is:

$$
\mathcal{C}(X)=\{A \subset X \mid A \text { is a continuum }\} .
$$

It is known that $\mathcal{C}(X)$ is a metric space with the Hausdorff metric $([\mathrm{N} 2,(0.1)])$, and in fact, $\mathcal{C}(X)$ is a continuum ([N2, (1.13)]).

Given a continuum $X$, the cone over $X$ is the decomposition space of the upper semicontinuous decomposition $(X \times[0,1]) / X \times\{1\}$. The cone over $X$ will be denoted by $\mathcal{K}(X)$, its base $X \times\{0\}$ by $\mathcal{B}(X)$, and its vertex $X \times\{1\} \in \mathcal{K}(X)$ by $v$. The symbol $\pi$ will denote the projection $\pi: \mathcal{K}(X) \backslash\{v\} \rightarrow \mathcal{B}(X)$ given by $\pi((x, t))=(x, 0)$.

Duda in $[\mathrm{D}]$ made a very interesting study of the hyperspaces of graphs, showing that $\mathcal{C}(X)$ is a finite-dimensional locally connected continuum if and only if $X$ is a graph, and that $\mathcal{C}(X)$ is a polyhedron if and only if $X$ is a graph.

From now on, we are going to assume that $X$ is a locally connected continuum for which $\operatorname{dim}(\mathcal{C}(X))<\infty$, hence $X$ is a graph (see [D, 1.1]).

We are going to use Duda's notation of [D], in particular we are going to assume that the graph $X$ satisfies the following conditions:

$(\alpha)$ : Each segment of $X$ has length equal to 1 and the distance between any two of its points is equal to the length of the shortest arc joining them.

$(\beta)$ : Each vertex of $X$ is either an end-point or a ramification point of $X$.

Given a graph $X, \mathcal{D}_{\mathcal{C}(X)}=\mathrm{Cl}_{\mathcal{C}(X)}\left\{C \in \mathcal{C}(X) \mid \operatorname{dim}_{C} \mathcal{C}(X)=2\right\}$, that is, $\mathcal{D}_{\mathcal{C}(X)}$ is the closure of the 2-dimensional part of the polyhedron $\mathcal{C}(X)$.

Given an arbitrary polyhedron $P$, by $\mathcal{E}_{P}$ we denote the closure (in $P$ ) of the subset of $P$ consisting of all points $p \in P$ for which there exists a closed neighborhood $V$ homeomorphic to a 2-dimensional ball and such that $p$ lies on the boundary of that ball (see Figure 1).

Duda showed the following two results:

Theorem $1([\mathrm{D}, 8.2])$. If $X$ is a graph, then $\mathcal{D}_{\mathcal{C}(X)}$ is homeomorphic to $X$ with a 2-dimensional ball attached to each segment $A$ of $X$ along an arc lying on the 


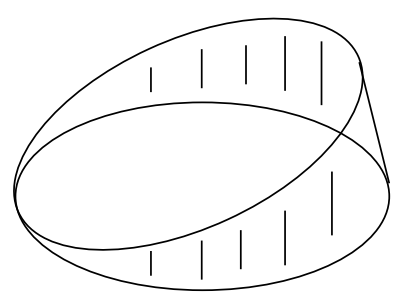

Figure 2

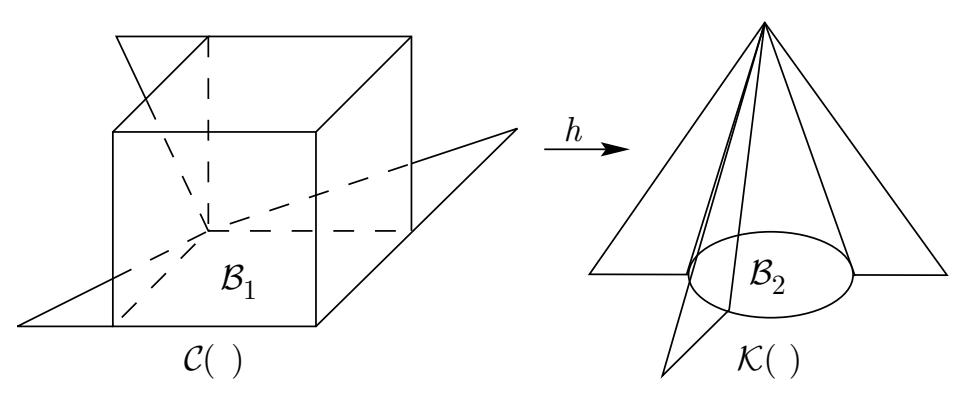

FiguRE 3

boundary of that ball in such a way that any two distinct balls are disjoint outside $X$.

Note that $\mathcal{D}_{\mathcal{C}(X)}$ consists of two types of "discs" $B$ : on one hand $B$ is just a triangle; on the other hand $B$ is a loop (a segment for which both end-points coincide) with a 2-dimensional ball attached along that loop (see Figure 2).

Theorem $2([\mathrm{D}, 8.4])$. If $X$ is a graph containing a ramification point, then $\mathcal{E}_{\mathcal{C}(X)}$ is homeomorphic to $X$.

\section{THE CHARACTERIZATION}

Theorem 3. If $X$ is a simple $n$-od, then $\mathcal{C}(X)$ is homeomorphic to the cone of a continuum $Z$.

Proof. Let $\mathcal{B}^{n-1}$ be the closed unit $(n-1)$-ball in $\mathbb{R}^{n-1}$ with center at the origin; let $A_{1}, \ldots, A_{n}$ be the straight line segments, which are mutually disjoint, from points $p_{1}, \ldots, p_{n}$ on the surface $\mathcal{S}^{n-2}$ of $\mathcal{B}^{n-1}$ to points $q_{1}, \ldots, q_{n}$ in $\mathbb{R}^{n-1} \backslash \mathcal{B}_{n-1}$. Then let $Z=\mathcal{B}^{n-1} \cup\left(\bigcup_{k=1}^{n} A_{n}\right)$.

Observe that $\mathcal{C}(X)$ and $\mathcal{K}(Z)$ have the same structure: each of them is the union of a convex $n$-ball and $n 2$-balls, which intersect on the surface of the $n$-ball in an $n$-od that is made up of $n$ convex arcs (see Figure 3 ). Denote the $n$-balls in $\mathcal{C}(X)$ and $\mathcal{K}(Z)$ by $\mathcal{B}_{1}$ and $\mathcal{B}_{2}$, respectively, and denote the $n$-ods in $\mathcal{C}(X)$ and $\mathcal{K}(Z)$ just mentioned by $N_{1}$ and $N_{2}$. Then, there is a homeomorphism, $h$, from $\mathcal{B}_{1}$ onto $\mathcal{B}_{2}$ such that $h\left(N_{1}\right)=N_{2}$. Clearly, $h$ can be extended to a homeomorphism from $\mathcal{C}(X)$ onto $\mathcal{K}(Z)$. Q.E.D.

Theorem 4. Let $X$ be a locally connected continuum whose hyperspace of subcontinua, $\mathcal{C}(X)$, has finite dimension. If $\mathcal{C}(X)$ is homeomorphic to the cone, $\mathcal{K}(Z)$, over a continuum $Z$, then $X$ is an arc, a simple closed curve, or a simple $n$-od. 
Proof. Suppose $\mathcal{C}(X) \underset{\text { top }}{=} \mathcal{K}(Z)$, and let $B$ be one of the 2-dimensional discs of $\mathcal{D}_{\mathcal{C}(X)}, B$ could be a loop with a 2 -dimensional ball attached along that loop. Let us observe that $B=\mathrm{Cl}_{\mathcal{C}(X)}\left(\operatorname{Int}_{\mathcal{C}(X)}(B)\right)$. Let $(z, t) \in \operatorname{Int}_{\mathcal{C}(X)}(B)$; then there exist an $\varepsilon>0$ and an open set $U$ of $\mathcal{B}(Z)$ such that $U \times(t-\varepsilon, t+\varepsilon) \subset \operatorname{Int}_{\mathcal{C}(X)}(B)$, thus, $U \times(t-\varepsilon, t+\varepsilon)$ is a 2 -dimensional open set of $\mathcal{C}(X)$, hence $U \times(0,1)$ is a 2 -dimensional open set of $\mathcal{C}(X)$ containing $\{(z, 0)\} \times(0,1)$. Since $\{(z, 0)\} \times(0,1)$ is connected and the 2-dimensional part of $\mathcal{C}(X)$ is contained in $\mathcal{D}_{\mathcal{C}(X)}$, by Theorem 1 , we have that $\{(z, 0)\} \times(0,1)$ is contained in $B$, otherwise $\{(z, 0)\} \times(0,1)$ would contain a ramification point $(z, s)$ of $\mathcal{E}_{\mathcal{C}(X)}$, but the dimension of $\mathcal{C}(X)$ at the point $(z, s)$ is bigger than two, a contradiction, hence $\{(z, 0)\} \times(0,1)$ is contained in $B$, in fact $\{(z, 0)\} \times(0,1) \subset \operatorname{Int}_{\mathcal{C}(X)}(B)$. Observe that this implies that $v \in B$. Let us note that $v$ is a ramification point of $\mathcal{E}_{\mathcal{C}(X)}$, otherwise $\mathcal{D}_{\mathcal{C}(X)}$ would consist of one disc, which would imply that $X$ would contain only one segment, that is, $X$ would be an arc or a circle, contrary to our hypothesis.

Now let $\mathcal{A}=\pi\left(\operatorname{Int}_{\mathcal{C}(X)}(B)\right)$; then $\mathcal{A}$ is an open, connected and locally arcwise connected subset of $\mathcal{B}(Z)$.

Note that $\pi(B \backslash\{v\})=\mathrm{Cl}_{\mathcal{B}(Z)}(\mathcal{A})$. To prove this, let $(z, 0) \in \pi(B \backslash\{v\})$; then there exists $t \geq 0$ such that $(z, t) \in B \backslash\{v\}$. Since $\operatorname{Int}_{\mathcal{C}(X)}(B)$ is dense, there is a sequence $\left\{\left(z_{n}, t_{n}\right)\right\}_{n=1}^{\infty} \subset \operatorname{Int}_{\mathcal{C}(X)}(B)$ converging to $(z, t)$, hence the sequence $\left\{\left(z_{n}, 0\right)\right\}_{n=1}^{\infty} \subset \mathcal{A}$ and converges to $(z, 0)$. Thus $(z, 0) \in \mathrm{Cl}_{\mathcal{B}(Z)}(\mathcal{A})$. To see the other inclusion, let $(z, 0) \in \mathrm{Cl}_{\mathcal{B}(Z)}(\mathcal{A})$; then there exists a sequence $\left\{\left(z_{n}, 0\right)\right\}_{n=1}^{\infty}$ contained in $\mathcal{A}$ and converging to $(z, 0)$. Let $0<t<1$, hence $\left\{\left(z_{n}, t\right)\right\}_{n=1}^{\infty}$ is contained in $\operatorname{Int}_{\mathcal{C}(X)}(B)$, thus $\left\{\left(z_{n}, t\right)\right\}_{n=1}^{\infty}$ converges to $(z, t) \in B \backslash\{v\}$ and $\pi((z, t))=(z, 0)$. Therefore $\mathrm{Cl}_{\mathcal{B}(Z)}(\mathcal{A})=\pi(B \backslash\{v\})$, and $B=\mathcal{K}\left(\mathrm{Cl}_{\mathcal{B}(Z)}(\mathcal{A})\right)$.

We will show that $\mathcal{E}_{\mathcal{C}(X)}$ contains only one ramification point. Suppose $\mathcal{E}_{\mathcal{C}(X)}$ has more than one ramification point. Let $v_{1}$ be a ramification point of $\mathcal{E}_{\mathcal{C}(X)}$ different from $v$. Since $v_{1}$ is a ramification point of $\mathcal{E}_{\mathcal{C}(X)}$, there are at least two 2-dimensional discs $B_{1}$ and $B_{2}$ of $\mathcal{D}_{\mathcal{C}(X)}$ such that $v_{1} \in B_{1} \cap B_{2}$ (condition $(\beta)$ in the Introduction). Then $\pi\left(v_{1}\right) \in \mathrm{Cl}_{\mathcal{B}(Z)}\left(\mathcal{A}_{1}\right) \cap \mathrm{Cl}_{\mathcal{B}(Z)}\left(\mathcal{A}_{2}\right)$, where $\mathcal{A}_{l}=\pi\left(\operatorname{Int}_{\mathcal{C}(X)}\left(B_{l}\right)\right)$, $l \in\{1,2\}$. Hence, we would have that $\pi\left(v_{1}\right) \times[0,1) \cup\{v\} \subset B_{1} \cap B_{2}$, but this contradicts the fact that $B_{1} \cap B_{2}$ contains at most two points, Theorem 1 .

Finally we will prove $\mathcal{E}_{\mathcal{C}(X)}$ does not contain loops. Suppose $\mathcal{E}_{\mathcal{C}(X)}$ has a loop $A$; then the disc $B$ of $\mathcal{D}_{\mathcal{C}(X)}$ containing $A$ would not be contractible. On the other hand, since $B=\mathcal{K}\left(\mathrm{Cl}_{\mathcal{B}(Z)}(\mathcal{A})\right)$, we would have that $B$ is contractible, a contradiction. Therefore $\mathcal{E}_{\mathcal{C}(X)}$ does not contain loops. Hence $\mathcal{E}_{\mathcal{C}(X)}$ is a graph with one ramification point and without loops, thus $\mathcal{E}_{\mathcal{C}(X)}$ is an $n$-od. Since $X$ is homeomorphic to $\mathcal{E}_{\mathcal{C}(X)}$, Theorem 2, we have that $X$ is an $n$-od. Q.E.D.

\section{REFERENCES}

[D] R. Duda, On the hyperspaces of subcontinua of a finite graph, I, Fund. Math., 62 (1968), 265-286. MR 38:5175a

[D-R] A. M. Dilks and J. T. Rogers, Jr., Whitney stability and contractible hyperspaces, Proc. Amer. Math. Soc. 83 (1981), 633-640. MR 82i:54023

[H-Y] J. Hocking and G. Young, Topology, Dover, 1988. MR 90h:54001

[I] A. Illanes, Hyperspaces homeomorphic to cones, Glasnik Mat. 30 (50) (1995), 285-294. MR 96k:54009

[N1] S. B. Nadler, Jr., Continua whose cone and hyperspace are homeomorphic, Trans. Amer. Math. Soc., 230 (1977), 321-345. MR 57:4126

[N2] S. B. Nadler, Jr., Hyperspaces of Sets, Marcel Dekker, 1978. MR 58:18330

[N3] S. B. Nadler, Jr., Continuum Theory, Marcel Dekker, 1992. MR 93m:54002 
[R1] J. T. Rogers, Jr., The cone-hyperspace property, Can. J.Math., 24 (1972), 279-285. MR 45: 4370

[R2] J. T. Rogers, Jr., Continua with cones homeomorphic to hyperspaces, Gen. Top. and Its Applications, 3 (1973), 283-289. MR 50:14699

Instituto de Matemáticas, Circuito Exterior, Ciudad Universitaria, México, D.F., C.P. 04510, MÉXICO

E-mail address: macias@servidor.unam.mx 